\title{
Germanica
}

\section{Une présentation : La dialectique de la raison de Max Horkheimer \& Theodor Adorno}

Uber Die Dialektik der Aufklärung von Max Horkheimer und Theodor Adorno

\section{Pierre Belaval}

\section{OpenEdition}

\section{Journals}

Édition électronique

URL : http://journals.openedition.org/germanica/2446

DOI : $10.4000 /$ germanica. 2446

ISSN : 2107-0784

Éditeur

Université de Lille

Édition imprimée

Date de publication : 31 décembre 1990

Pagination : 195-202

ISBN : 9782913857025

ISSN : 0984-2632

\section{Référence électronique}

Pierre Belaval, « Une présentation : La dialectique de la raison de Max Horkheimer \& Theodor Adorno », Germanica [En ligne], 8| 1990, mis en ligne le 28 novembre 2014, consulté le 06 octobre 2020. URL http://journals.openedition.org/germanica/2446 ; DOI : https://doi.org/10.4000/germanica.2446

Ce document a été généré automatiquement le 6 octobre 2020.

(ㄷ) Tous droits réservés 


\title{
Une présentation : La dialectique de la raison de Max Horkheimer \& Theodor Adorno ${ }^{1}$
}

Uber Die Dialektik der Aufklärung von Max Horkheimer und Theodor Adorno

\author{
Pierre Belaval
}

1 L'ouvrage de Max Horkheimer (1875-1973) et de Theodor Adorno (1903-1969), achevé en 1944, et publié en 1947 à Amsterdam, est considéré comme l'un des plus représentatifs de l'École de Francfort. Celle-ci, fondée en 1923 à Francfort sous le nom d'Institut für Sozialforschung, se replia en 1933 à Genève puis transféra ses fonds aux États Unis en 1941 pour revenir à Francfort en 1950. La «théorie critique » qu'elle construisit en ses débuts visait déjà à mettre en lumière les mobiles sociaux cachés de la «théorie traditionnelle ", c'est-à-dire de la théorie scientifique comme mise en ordre des faits visant l'exactitude, et cherchait par une critique de la science liée à celle de la société à instituer, par le biais de la révolution, une société meilleure ${ }^{2}$. Or cette espérance se trouve ruinée après l'expérience du fascisme, du national-socialisme et du stalinisme. Fallait-il alors tomber dans l'irrationalisme? Il s'agit, au contraire, de repenser totalement le statut de la raison occidentale, de conduire, comme le montre Jürgen Habermas ${ }^{3}$, une instruction de la raison sur elle-même pour voir comment celle-ci s'est transformée en une raison instrumentale, voire même s'est autodétruite. C'est le procès d'une conception naïve du progrès comme triomphe des lumières de la raison sur un passé mythique définitivement révolu qui est ici instruit. Il s'agit de mettre à jour une complicité secrète entre mythe et Lumières, le terme de Lumières n'étant pas pris ici au sens étroit de la philosophie des Lumières du XVIII ${ }^{e}$ siècle, mais pris au sens large de "pensée en progrès", voire "philosophie du progrès». Comment penser philosophiquement à la fin de la Seconde guerre mondiale l'effondrement de la civilisation bourgeoise comme éclipse de la raison et régression de celle-ci vers les mythologies?

2 La Dialectique de la raison ne présente pas une unité systématique, mais regroupe trois études suivies de quelques remarques marginales. La première intitulée «Le concept 
d'Aufklärung " veut "faire comprendre l'intrication de la rationalité et de la réalité sociale, ainsi que l'intrication de la nature et de la domination de la nature qui en est inséparable " (D.R., p. 18). Elle se réduit à deux thèses que Habermas considère comme les deux thèses centrales du livre : « le mythe est déjà raison et la raison se retourne en mythologie » (D.R., p. 18). Elle est complétée par deux digressions, l'une sur l'Odyssée comme première figure de l'avènement de la raison, l'autre sur Kant, Sade et Nietzsche conduisant la raison jusqu'à la limite de ses implications. La seconde étude intitulée "La production industrielle des biens culturels : raison et mystification des masses " " analysera la régression de la raison dans une idéologie contrôlée par la technique et la prétention de ces biens culturels à se constituer comme objet esthétique. La troisième étude intitulée; "Éléments de l'antisémitisme - limites de la raison» analysera le retour de la civilisation actuelle à la barbarie.

Nous présenterons ici les données essentielles de ces analyses et les confronterons à la lecture critique qu'en fit J. Habermas dans la cinquième partie de son œuvre Le Discours philosophique de la modernité.

La première étude commence par rappeler les ambitions de l'Aufklärung: libérer l'homme des peurs de la magie et du mythe par un savoir et un pouvoir sans limites, dont l'essence est technique, et en finir avec l'animisme. Sur cette voie, qui la conduit à la science moderne, la raison « renoncerait au sens, et remplacerait le concept par la formule, la cause par la règle et la probabilité ». Tout ce qui ne se conforme pas aux critères du calcul et de l'utilité devient suspect à la raison (D.R., p. 24). Celle-ci prétend mettre en équation non seulement la nature, mais le monde socio-économique. Cette science arrivant à maturité développe une confiance inébranlable en ses possibilités de dominer le monde, « se comporte à l'égard des choses comme un dictateur à l'égard des hommes, qui ne les connaît que dans la mesure où il peut les manipuler» (D.R., p. 27). Or cette entreprise conquérante de la raison instrumentale va produire son propre contraire : devenir une entreprise de liquidation de la civilisation. Elle détruit la pensée symbolique du mythe et ses successeurs, les concepts de la métaphysique, pour penser la nature comme ce qui doit être appréhendé mathématiquement. Et elle se croit ainsi à l'abri du mythe. Mais, en fait, elle y retourne en s'appropriant la réalité comme un schéma mathématique et en la répétant comme tel. Les anciens démons des mythologies reviennent sous des formes nouvelles: l'homme est aliéné à ce qu'il produit, l'esprit de même que les relations entre les hommes modelant leurs corps et leurs âmes sur leurs équipements techniques sont réifiés, la marchandise est fétichisée, la crainte d'une nature incontrôlée et menaçante se développe. "L'animisme avait donné une âme à la chose, l'industrialisme transforme l'âme de l'homme en chose " (D.R., p. 44) et ainsi les hommes redeviennent semblables aux rameurs du bateau d'Ulysse, obéissant tous au même rythme et à des conditions de travail appelant au conformisme.

4 Cette première étude est illustrée par la digression II : Ulysse ou mythe et raison. L'Odyssée toute entière et l'univers homérique témoignent de cette dialectique de la raison. Ils constituent un produit de la raison organisatrice détruisant le mythe par un ordre rationnel et décrivant la fuite du sujet individuel devant les puissances mythiques. Tout comme Ulysse se perd pour se retrouver, le Moi n'atteint son unité qu'à travers la diversité de ses aventures. Le sacrifice aux puissances mythiques 
devient, par Ulysse, stratagème. La ruse d'Ulysse est avènement d'une raison "bourgeoise " exigeant la lucidité, le sens des réalités, évaluant avec précision les rapports de force, défi rationalisé aux puissances du destin. Mais tout en restant distant de la nature, Ulysse écoute encore sa voix: Ulysse est donc la figure de l'homme renonçant à l'unité archaïque avec la nature et apprenant à la dominer au prix d'une répression de sa propre nature. C'est à la lumière de ce fil directeur qu'Adorno et Horkheimer interprètent différents passages de l'Odyssée : l'épisode des mangeurs de lotus, la lutte avec Polyphème, l'épisode de Circé, le séjour dans l'Hadès.

5 La digression II s'intitule Juliette ou raison et morale. Adorno et Horkheimer partent du célèbre texte de Kant de 1784 : Was ist Aufklärung? où l'Aufklärung - cette fois au sens étroit - est définie comme la sortie de l'homme d'un état de minorité dont il est luimême responsable, c'est-à-dire d'une incapacité de se servir de son propre entendement sans la direction d'autrui. Selon l'étonnante interprétation des textes kantiens proposée par Adorno et Horkheimer, l'entendement doit être dominé par la raison qui veut penser un ordre scientifique unitaire venant à bout des faits. La raison est comprise comme instance d'un penser calculateur qui organise le monde en vue d'une conservation de soi. La "véritable » nature du schématisme des concepts purs de l'entendement est d'exprimer l'intérêt de la société industrielle appréhendée sous l'aspect de la manipulation et de l'administration, et la définition kantienne du devoir comme nécessité d'agir par respect pour la loi morale est interprétée comme "tentative bourgeoise de donner au respect, sans lequel la civilisation ne saurait exister, des fondements autres que l'intérêt matériel et la violence ».

Que va devenir dans l'interprétation d'Horkheimer et Adorno le projet d'un entendement non dirigé par un autre? Il se manifeste sous les traits du bourgeois libéré de toute tutelle que nous montre l'œuvre de Sade. La raison devient organe de planification et de calcul, neutre à l'égard des buts, création de mythologies nouvelles taxant de superstition les dévotions précédentes. Justine, la sœur vertueuse de Juliette, devient martyre de la loi morale tandis que Juliette démonise le christianisme, détruit de manière systématique et cohérente la civilisation avec ses propres armes. La mission de Juliette anticipe alors déjà certains thèmes nietzschéeens: la transvaluation de toutes les faibles et les ratés, le désir de vivre dangereusement. Injustice et haine deviennent des activités systématiques et la domination devient jouissance devant le désespoir total de la victime. Ainsi « les vices privés chez Sade comme chez Mandeville sont l'historiographie anticipée des vertus publiques des sociétés totalitaires » (D.R., p. 127).

7 La seconde étude va se centrer sur un examen critique des secteurs de la culture, que la civilisation actuelle uniformise et standardise tant au niveau des publics que des œuvres : ceci conduit à un art sans rêve, paralysant l'imagination et la réflexion, à une rigidité du style, un mépris des connaisseurs et des experts, une culture de masse excluant toute nouveauté comme risque inutile, une industrie culturelle ne nourrissant les hommes que de stéréotypes. L'industrie culturelle instaure le règne de la fausse individualité, où, par une répétition mécanique, les produits culturels sont traités comme des slogans politiques. Par le caractère publicitaire et la langue, s'opère également un retour à la mythologie : le mot se rive à la chose, il devient une « formule pétrifiée » et les hommes eux-mêmes sont réifiés. Ces éléments seront développés dans d'autres études d'Adorno ${ }^{4}$ et, bien sûr, dans l'« homme unidimensionnel » de Marcuse. 
8 La dernière étude se situe dans la ligne des travaux de l'École de Francfort utilisant les catégories psychanalytiques comme instruments d'une critique des idéologies. Elle s'intitule : Éléments de l'antisémitisme. Deux doctrines s'affrontent ici : celle des libéraux considérant les juifs comme une minorité, un groupe fondé sur ses opinions et traditions religieuses, celle des fascistes les considérant comme le mal absolu et projetant sur eux leur propre nature, l'avidité d'une possession exclusive et d'un pouvoir illimité. La thèse libérale construit l'image d'une société où la haine cesse de se produire et où les juifs, en étant assimilés, surmontent les souvenirs douloureux de la persécution. Mais l'harmonie de cette société va se retourner contre les minorités sous la forme d'une Volksgemeinschaft les persécutant.

Horkheimer et Adorno veulent montrer l'échec des explications politiques et économiques de l'antisémitisme. Celui-ci joue selon leur interprétation, le rôle d'un meurtre rituel, la fonction d'une soupape de sécurité où la haine se décharge sur des victimes sans défenses, des sortes de boucs émissaires de l'injustice économique. L'antisémitisme raciste ignore les considérations d'ordre religieux, et se préoccupe de la pureté de la «race » et de la "nation ». Il reproduirait des attitudes renvoyant aux origines : attitude défensive ou de raidissement due à la faveur et la simple existence de l'autre devient une véritable provocation. Ainsi le Juif ne doit connaître aucun repos, même dans la mort (cf. les profanations par les nazis des cimetières juifs). Le fascisme en revient à des comportements mimétiques favorisés par la discipline, les rituels des discours et des fêtes, et réhabilite l'horreur de la préhistoire, écrasée par la civilisation, dans la persécution des juifs. L'antisémitisme est ainsi pensé comme fausse projection d'impulsions que le sujet n'admet pas comme siennes sur une victoire potentielle, incapacité de réfléchir sur l'objet et sur soi-même, véritable pathologie du penser dans un excès de cohérence et une immobilité, « trait propre à toute mentalité acceptant les étiquettes » (D.R., p. 215).

10 Tel est l'aboutissement extrême de cette dialectique de la raison, qui, par différence avec la dialectique hégélienne, ne fait point place à une "Aufhebung " réconciliant l'esprit avec lui-même, le penser et l'être, mais reste une dialectique négative logique de la dislocation devant rendre compte d'une perversion essentielle de la raison que cette dialectique négative déchiffrera dans trois faits contemporains: Auschwitz, impensable comme simple accident ou déviation de l'histoire face à une dynamique de l'Aufklärung ${ }^{5}$, les aventures de la dialectique marxiste dans le stalinisme, l'avènement enfin dans les sociétés occidentales d'un « homme unidimensionnel ».

11 Il est alors intéressant d'examiner avec Habermas les motivations qui ont conduit Horkeimer et Adorno à une critique aussi radicale. L'Aufklärung apparaît en effet comme une « tentative désespérée pour échapper à l'origine que sont les puissances du destin » (D.R.M., p. 137) et "le vide désolant sur lequel débouche l'émancipation est la forme sous laquelle la malédiction des puissances mythiques finit par frapper les fugitifs» (idem.). Dire que le processus de l'Aufklärung a désocialisé la nature et dénaturalisé le monde humain n'est pas seulement reprendre le thème wébérien d'un désenchantement du monde, mais montrer un décentrement de sa conception. Il ne s'agit pas simplement ici d'une répétition de la critique marxiste des idéologies ou du fruit de déceptions historiques liées à la victoire du fascisme et du stalinisme, mais 
d'une radicalisation et d'une surenchère dans la critique de la raison soupçonnée de confondre prétention au pouvoir et prétention à la validité. D'où, pour Habermas, le paradoxe d'une telle description de l'autodestruction subie par la faculté de critique dont elle proclame la mort. Habermas établit alors une comparaison avec la critique nietzschéenne de la culture, notamment dans La Généalogie de la morale, où les hommes, pris dans le carcan de la paix et de la société, doivent renoncer à extérioriser leurs instincts, se fier à leur conscience et se laisser domestiquer par une domination de la nature externe et de leur nature interne. On sait également que l'entreprise nietzschéenne prétend démasquer le caractère fictif des idéaux de la raison théorique ou pratique mettant à jour des forces réactives, symptômes d'une volonté de puissance épuisée, opérant secrètement en eux. Par le biais d'une critique généalogique, Nietzsche développerait le thème d'une culture véritable ayant disparu depuis longtemps où le plus ancien est le plus distingué, l'expression de forces actives et affirmatives de la vie tandis que les formes de cultures plus tardives ne seraient que les masques d'une volonté de puissance pervertie. Le problème n'est-il pas analogue pour Horkheimer et Adorno ? Comment poursuivre - se demande Habermas - le travail de critique sans préserver de la corruption un critère rationnel? Nietzsche se réfugiait dans une théorie du pouvoir analysant les forces affirmatives de la vie ou réactives de la volonté de puissance. Horkheimer et Adorno tombent dans un «scepticisme effréné vis-à-vis de la raison au lieu d'examiner les raisons qui permettent de douter de ce scepticisme même " (D.P.M., p.155) et confondent les lumières de la découverte, la connaissance qu'on apporte, avec les justifications qu'on en donne. Telles sont la limite et la faiblesse de cette critique de la raison instrumentale ${ }^{6}$.

\section{NOTES}

1. - Titre original Die Dialektik der Aufklärung-philosophische Fragmente, C Social studies Assoc. Inc., New-York, 1944, C.S. Fischer Verlag Gmbh, Frankfurt/Main, 1969, pour la nouvelle édition, éditée également dans la collection de poche Fischer Taschenbücher $\mathrm{Nr} 6144$; traduction française par Éliane Kaufholz, bibliothèque des Idées, Gallimard, 1974 et repris par le même éditeur dans la collection « Tel ». Les citations faites renvoient à cette traduction sous le titre abrégé D.R.

2. - Cf. l'article de Horkheimer écrit à la mort d'Adorno intitulé : «Théorie critique hier et aujourd'hui » et paru dans Théorie critique, traduction française de Payot, 1978, p. 354-369.

3. - Titre original: Der philosophische Diskurs der Moderne-12 Vorlesungen, Suhrkamp Verlag, Frankfurt/Main, 1985, traduit en français sous le titre: "Le discours philosophique de la modernité - douze conférences" par Christian Bouchindomme et Xavier Rochlitz dans la collection «bibliothèque de philosophie", 1988, $5^{\mathrm{e}}$ partie, p. 128-156. Les citations faites renvoient à cette traduction sous le titre abrégé D.R.M.

4. - Par exemple dans Adorno, Minima moralia, trad. française Payot, 1980, ainsi que Modèles critiques, trad. française Payot, 1984, ou Prismes, trad. française Payot, 1986.

5. - Cf. l'article d'Adorno «Éduquer après Auschwitz » paru dans Modèles critiques, Payot, 1984, p. 205-220. 
6. - Pour avoir une vue d'ensemble sur l'École de Francfort, on peut lire d'une part l'ouvrage de Martin Jay, L'imagination dialectique. L'École de Francfort, Payot et le petit ouvrage très clair de PaulLaurent Assoun, L'École de Francfort, P.U.F., 1986, collection « Que sais-je ?», n²354.

\section{RÉSUMÉS}

Dans cet ouvrage composé à la fin de la seconde guerre mondiale, Horkheimer et Adorno proposent une interprétation originale de l'effondrement de la civilisation occidentale. Les idéaux de la raison et du progrès n'ont pas laissé le mythe et la barbarie loin derrière eux, mais ont produit leur propre contraire : une éclipse, voire une autodestruction de la raison, réduite à une raison instrumentale planificatrice, qui ne s'interroge plus sur les fins qu'elle met en jeu. Nous analyserons, à travers les trois études qui constituent ce livre, le développement de ses deux thèses centrales : le mythe est déjà raison, et la raison retourne au mythe, et nous les confrontons à l'examen critique qu'en fait Jürgen Habermas dans son œuvre Le Discours philosophique de la modernité.

In diesem im Jahre 1944 geschriebenen Buch wollen Adorno und Horkheimer den Zusammenbruch der abendländischen Kultur vom philosophischen Standpunkt aus ergründen. Man glaubt gewöhnlich, dass die Aufklärung die Barbarei und den Mythos zerstört hat. Hier behaupten Adorno und Horkheimer, dass der Kampf der Aufklärung für Freiheit, Gerechtigkeit und Humanität gerade zu ihrem Gegenteil geführt hat, und dass die Vernunft ein blosses technisches Instrument geworden ist. So hat sich die Aufklärung selbst zerstört. Wir werden hier die zwei Grundideen dieses Buches: «schon der Mythos ist Aufklärung und Aufklärung schlägt in Mythologie zurück » analysieren und sie mit der Kritik von Jürgen Habermas in seinem Werk: «Der philosophische Diskurs der Morderne, 12 Vorlesungen» konfrontieren.

\section{AUTEUR}

\section{PIERRE BELAVAL}

Lycée Pasteur, Lille 